\title{
Reconsideration of pathology and management for acute antibody- mediated rejection in pediatric ABO incompatible transplantation
}

\author{
Yusuke Yanagi, Seisuke Sakamoto, Kotarou Mimori, Masatoshi Nakao, Seiichi Shimizu, Hajime Uchida, Akinari Fukuda,
} Mureo Kasahara

Organ Transplantation Center, National Center for Child Health and Development, Tokyo, Japan

Background: The management and outcome of ABO incompatible liver transplantation (ABO-I LT) has been improved in the decades. Recently, latest pathological evaluation of acute antibody-mediated rejection (AMR) was introduced through Banff meeting and provides a new aspect.

Methods: One hundred nineteen pediatric ABO-I LT performed in our institute from 2005 to 2020 were retrospectively analyzed. All specimens of liver biopsy were newly evaluated by the Banff 2016 criteria. We evaluated the clinical and pathological differences of acute AMR according to the patient age based on the need for preoperative rituximab (RTx): the indication of preoperative RTx was older than 2 years until 2016 and changed to 1.5 years from 2017.

Results: Fourteen acute AMR related to ABO-I LT was observed. The median age of the patients with acute AMR was 2.4 years and the youngest age was 8 months. Eleven cases were mixed T cell-mediated rejection (TCMR)/AMR indicating the synergic action between humoral and cellular rejections. Two young patients in early era were diagnosed as TCMR at that time, then revealed as mixed TCMR/AMR in this study. Isolated AMR were observed in three patients in the older group requiring RTx, who were not administered preoperative RTx appropriately. Six out of seven patients with acute AMR in the older group required plasma exchange (PE) and one patient required anti-thymocyte immunoglobulin (ATG) for treatment. However, except for one case suffered from intrahepatic biliary complication treated with PE, ATG, and RTx, six patients with mixed TCMR/AMR in the younger group omitted RTx were successfully treated with steroid bolus injection, intravenous immunoglobulin and mycophenolate mofetil without PE.

Conclusions: Acute AMR in pediatric ABO-I LT can often accompany TCMR, even in children younger than 1 year. It should be actively diagnosed with pathology and treated by anti-B cell strategy, combined with anti-T cell strategy if needed.

Corresponding author: Yusuke Yanagi

E-mail: yanagi-y@ncchd.go.jp

(C) The Korean Society for Transplantation

This is an Open Access article distributed under the terms of the Creative Commons Attribution Non-Commercial License (http://creativecommons.org/licenses/by-nc/4.0/) which permits unrestricted non-commercial use, distribution, and reproduction in any medium, provided the original work is properly cited. 